Comprendre la manière

dont un matériau composé

de grains s'écoule a des

répercussions dans des situa-

tions pratiques très variées :

transport de sable ou de graviers dans le génie civil, mélange

de poudres dans les industries

pharmaceutiques et cosmétiques,

stockage de céréales dans

le secteur agroalimentaire.

Dans la nature, la formation de cratères d'impacts météoritiques

ou encore la locomotion

animale dans les zones

désertiques sont des

exemples parmi d'autres où

des matériaux granulaires

s'écoulent autour d'un obstacle.

Une approche de type

" hydrodynamique » permet-

elle de décrire ces écoulements

de grains ? En quoi sont-ils

fondamentalement différents

d'un fluide simple?

\title{
Plongée dans le sable
}

Yann Bertho (1) (yann.bertho@u-psud.fr), Antoine Seguin ${ }^{(1)}$, Philippe Gondret ${ }^{(1)}$ et Jérôme Crassous ${ }^{2}$ (1) Laboratoire FAST, Université Paris-Sud, CNRS (UMR 7608), 91405 Orsay Cedex

(2) Institut de Physique de Rennes, Université de Rennes 1, CNRS (UMR 6251), 35042 Rennes

La difficulté d'appréhender un écoulement de grains réside non seulement dans la résolution de problèmes d'hydrodynamique, comme pour les fluides usuels dits newtoniens (encadré 1), mais aussi dans le choix des équations de comportement du "fluide granulaire ". Comme pour les fluides classiques, on pourra définir une notion de température pour ces milieux granulaires : l'agitation (ou fluctuations de vitesse) des grains permet de déterminer une température granulaire, par analogie avec la température thermodynamique classique liée à l'agitation moléculaire. Une différence essentielle réside néanmoins dans le caractère fortement dissipatif de ces matériaux, dû notamment aux collisions entre grains.

Afin de caractériser ces écoulements granulaires autour d'objets, des expériences récentes réalisées au laboratoire FAST à Orsay consistent à faire pénétrer à vitesse constante un objet cylindrique de quelques centimètres de diamètre dans un matériau granulaire modèle, constitué de petites billes millimétriques (fig. 1a). Des prises de vue successives des grains autour de l'objet à travers la paroi vitrée permettent, par une technique de corrélation d'images, d'accéder dans son référentiel au champ de vitesse moyenne des grains et à ses fluctuations
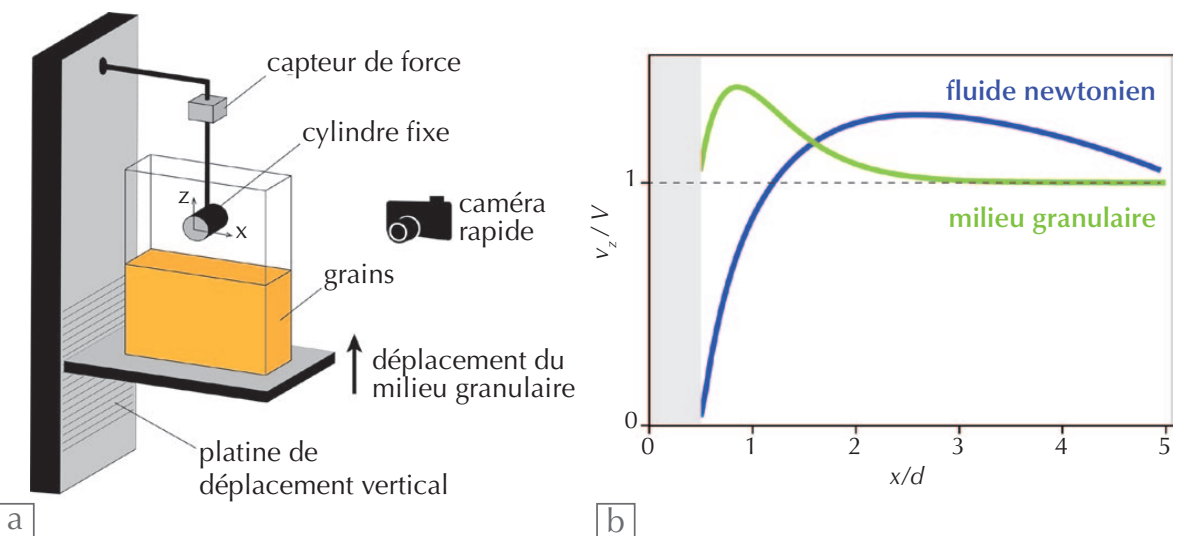

$\mathrm{b}$

temporelles durant toute la pénétration (fig. 2). Un capteur de force relié au cylindre permet également de mesurer l'évolution de la force au cours de sa pénétration.

Les résultats observés sont très différents de ceux obtenus avec des fluides classiques newtoniens. En effet, sur trois décades de vitesse explorées $V$ (de 0,1 à $100 \mathrm{~mm} / \mathrm{s}$ ), les profils de vitesse moyenne des grains sont stationnaires avec une forte localisation de la vitesse au voisinage immédiat de l'objet (fig. 1b). La survitesse de part et d'autre du cylindre est suivie d'une décroissance rapide avec un point d'inflexion et une asymptote horizontale, soulignant qu'à une distance à peine supérieure au diamètre du cylindre, les grains ne sont déjà plus perturbés par sa présence. Pour un fluide visqueux newtonien en revanche, la perturbation aurait une portée beaucoup plus longue, jusqu'aux parois du récipient ! Notons également que les grains glissent à la paroi du cylindre, alors qu'il y a classiquement une condition de non-glissement pour un fluide newtonien. Par ailleurs, la force ressentie par le cylindre ne dépend pas de la vitesse de déplacement, contrairement à la force de trainée dans un fluide visqueux, proportionnelle à la vitesse.

1. (a) Schéma du dispositif expérimental permettant l'observation du mouvement vertical d'un cylindre dans un milieu granulaire.

(b) Profil de la composante verticale $v_{z}$ de la vitesse des grains, adimensionnée par la vitesse de pénétration $V$, suivant l'horizontale $z=0$, passant par l'axe du cylindre (situé en $x=0$ ) de diamètre $d=20 \mathrm{~mm}$. La ligne en pointillés $\left(v_{2} / V=1\right)$ correspond à la valeur de vitesse non perturbée par la présence du cylindre. 
Si l'on s'intéresse maintenant aux fluctuations temporelles de la vitesse autour de la vitesse moyenne (i.e. la température granulaire), on constate que celles-ci sont importantes. Deux régions distinctes sont mises en évidence (fig. 2) : une couronne très localisée autour de l'objet, dans laquelle l'agitation des grains est très forte (fluctuations de l'ordre de $20 \%$ de la vitesse) et au-delà, une région dans laquelle la température granulaire décroît exponentiellement. Dans la couronne proche de l'objet, la température granulaire est à peu près constante, proportionnelle au carré de la vitesse de l'objet et inversement proportionnelle à son diamètre. Elle découle d'un équilibre entre d'une part une production de " chaleur " résultant du cisaillement près de l'objet et, d'autre part, une dissipation d'énergie par collision à l'échelle des grains. Cette forte agitation au voisinage immédiat de l'objet influe sur la fluidité du matériau, créant une zone d'écoulement facilité. De plus, un point remarquable est que la taille de cette couronne où l'écoulement des grains est privilégié, est indépendante de la vitesse de déplacement de l'objet dans le milieu granulaire et de la profondeur d'enfoncement! Elle dépend essentiellement du diamètre de l'objet et peu de la taille des grains.

Ainsi, dans une vision hydrodynamique, le déplacement d'un objet dans un milieu granulaire s'apparente au mouvement d'un objet chaud dans un fluide dont la viscosité dépend de la température. Cette situation correspond, par exemple, au cas géophysique de remontée de magma dans le manteau terrestre : on y retrouve un écoulement très localisé au voisinage de ces "bulles magmatiques chaudes ", dans une région où la viscosité du manteau est fortement abaissée par diffusion de la chaleur. Cette vision hydrodynamique ouvre des perspectives pour aborder des situations plus complexes d'écoulements de grains, dans des contextes géophysiques ou industriels.
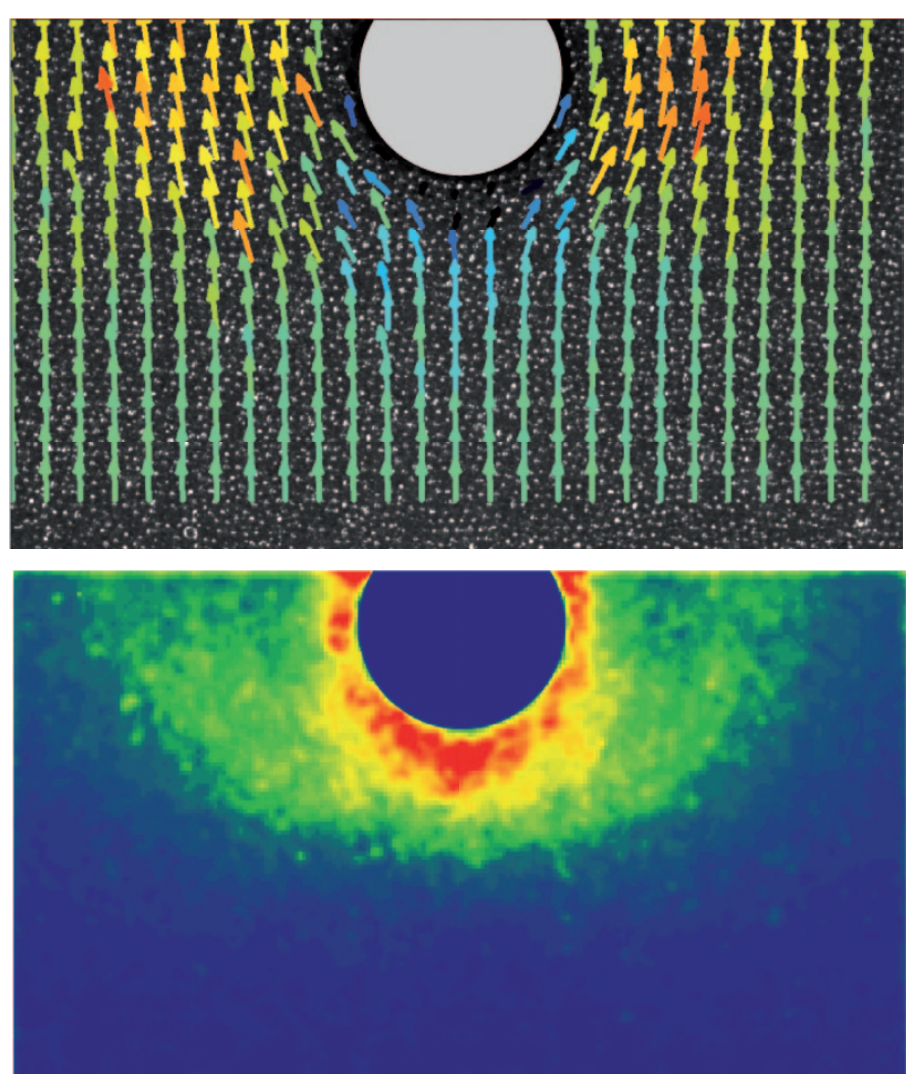

2. Champs de vitesse et de « température » d'un milieu granulaire s'écoulant autour d'un cylindre de $20 \mathrm{~mm}$ de diamètre, obtenue par vélocimétrie par images de particules (PIV).

En haut : le champ de vitesse des grains est perturbé dans le voisinage immédiat de l'objet, avec une survitesse de part et d'autre du cylindre, du bleu pour les faibles vitesses au rouge pour les plus fortes vitesses.

En bas : les températures granulaires élevées apparaissent en rouge, localisées dans une région proche de l'objet où le cisaillement est important, et les faibles températures apparaissent en bleu.

\section{Fluide newtonien}

Lorsqu'un fluide est cisaillé entre une paroi fixe et une paroi mobile de vitesse $V$ distantes de $H$, il existe au sein du fluide des contraintes tangentielles $\tau$ d'origine visqueuse qui dépendent du gradient de vitesse $\dot{\gamma}=V / H$.

Un fluide, comme l'eau par exemple, sera dit newtonien si les contraintes visqueuses sont proportionnelles au gradient de vitesse. Le coefficient de proportionnalité, noté ici $\eta$, est appelé la viscosité du fluide.

Tous les fluides s'écartant de cette loi sont dits non newtoniens, avec des comportements qui peuvent être très variés : fluides à seuil (pâte dentifrice), rhéofluidifiants (peinture), rhéoépaississants (Maïzena $\left.{ }^{\circledR}\right)$, etc.
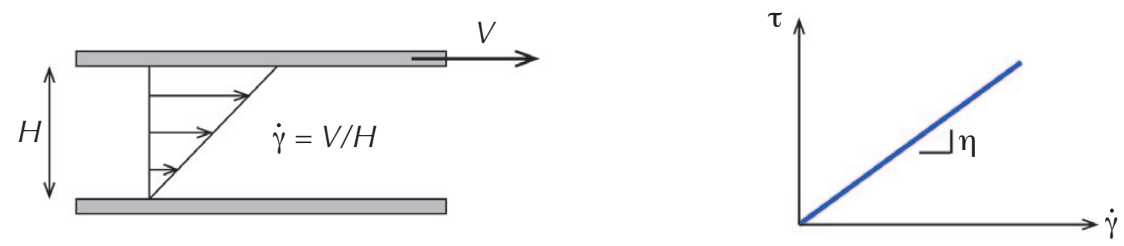

\section{Références}

1• A. Seguin et al., "Dense granular flow around a penetrating object: Experiment and hydrodynamic model", Phys. Rev. Lett. 107 (2011) 048001. 